\title{
c-Jun N-terminal Kinase (JNK) induces phosphorylation of amyloid precursor protein (APP) at Thr668, in okadaic acid-induced neurodegeneration
}

\author{
Ji-Hwan Ahn ${ }^{1,2,3,4, \#}$, Sang-Pil So ${ }^{1,2,3,4, \#}$, Na-Young Kim ${ }^{1,2,3,4, \#}$, Hyun-Ju Kim ${ }^{1,2,3,4}$, Seung-Yong Yoon ${ }^{1,2,3,4, *}$ \\ E Dong-Hou Kim ${ }^{1,2,3,4, *}$ \\ ${ }^{1}$ Alzheimer's Disease Experts Lab (ADEL), Asan Medical Center, University of Ulsan College of Medicine, ${ }^{2}$ Department of Brain Science, \\ ${ }^{3}$ Bio-Medical Institute of Technology (BMIT), ${ }^{4}$ Cell Dysfunction Research Center (CDRC), University of Ulsan College of Medicine, Seoul \\ 05505, Korea
}

Several lines of evidence have revealed that phosphorylation of amyloid precursor protein (APP) at Thr668 is involved in the pathogenesis of Alzheimer's disease (AD). Okadaic acid (OA), a protein phosphatase-2A inhibitor, has been used in AD research models to increase tau phosphorylation and induce neuronal death. We previously showed that OA increased levels of APP and induced accumulation of APP in axonal swellings. In this study, we found that in OA-treated neurons, phosphorylation of APP at Thr668 increased and accumulated in axonal swellings by c-jun $\mathrm{N}$-terminal kinase (JNK), and not by Cdk5 or ERK/MAPK. These results suggest that JNK may be one of therapeutic targets for the treatment of $A D$. [BMB Reports 2016; 49(7): 376-381]

\section{INTRODUCTION}

Alzheimer's disease (AD) is one of the most common neurodegenerative disorders, characterized by two pathologic hallmarks: neurofibrillary tangles (NFTs) and senile plaques (1). The extracellular senile plaques are primarily composed of $\beta$-amyloid peptides $(A \beta)$, which are derived from the amyloid precursor protein (APP) through intracellular proteolytic cleavages (2). On the other hand, NFTs are intraneuronal aggregations of paired helical filaments comprised of abnormally hyperphosphorylated tau proteins (3). Although

*Corresponding authors. Seung-Yong Yoon, Tel: +82-2-3010-4241; Fax: +82-2-3010-8038; E-mail: ysy@amc.seoul.kr, Dong-Hou Kim, Tel: +82-2-3010-4241; Fax: +82-2-3010-8038; E-mail: dhkim@ amc.seoul.kr

${ }^{\#}$ These authors contributed equally to this work.

http://dx.doi.org/10.5483/BMBRep.2016.49.7.246

Received 26 November 2015, Revised 14 December 2015, Accepted 3 February 2016

Keywords: Alzheimer's disease, APP, JNK, Okadaic acid, PP2A the precise relationships underlying these pathologic features are unclear, several lines of evidence demonstrate that the number of NFTs in the cortex of AD patients, are correlated with the duration and the severity of dementia (1-3). Given the positive relationship between NFTs and the severity of illness, aberrant phosphorylation of tau seems to be a critical step in the progress of neurodegeneration in AD. Moreover, the involvement of decreased protein phosphatase activity has been hypothesized to cause the neurofibrillary pathology of $A D$, though the specific cellular events that result in tau phosphorylation are not yet clear (4).

Okadaic acid (OA), a cytotoxin isolated for the first time from two marine sponges of the genus Halichondria (5), is a potent inhibitor of protein phosphatase-2A. It induces hyperphosphorylation of tau, and neuronal death in neuronal cultures (6). In rats, OA also gives rise to increased deposition of $A \beta$ followed by subsequent loss of neurons, reduced synaptic density, and memory impairment, all of which mimic characteristics of AD-like pathology (7). However, although these experimental paradigms are not sufficient to fully reconstitute the pathology of $A D$, they provide potentially useful tools for studying the participation of tau phosphorylation in the processes of neurodegeneration $(7,8)$.

APP is sequentially processed by at least three proteases, namely $\alpha-, \beta$-, and $\gamma$-secretases, that result in the generation of $A \beta$. Dysfunction in APP metabolism has been reported to increase $A \beta$ critical to $A D$ (9). Among multiple phosphorylation sites of APP, phosphorylation on the threonine residue of the VT668PEER motif (Thr668) within the intracellular domain of APP has been reported to be increased in human AD brains. This suggests that Thr668 phosphorylation may facilitate $\beta$-secretase cleavage of APP, and contribute to the generation of $A \beta(10)$. However, little is known about the exact mechanisms concerning the accumulation of $A \beta$ following phosphorylation of APP at Thr668. This is also a controversial theory, since that Sano et al. argue that $A \beta$ levels are not related to the phosphorylation state of Thr668 of APP (11).

Phosphorylation of APP at Thr668 is mediated by various

ISSN: 1976-670X (electronic edition)

Copyright (C) 2016 by the The Korean Society for Biochemistry and Molecular Biology

(c) This is an open-access article distributed under the terms of the Creative Commons Attribution Non-Commercial License (http://creativecommons.org/licenses/by-nc/4.0) which permits unrestricted non-commercial use, distribution, and reproduction in any medium, provided the original work is properly cited. 
protein kinases, such as GSK33, SAPK1b/JNK3, JNK1/2 and Cdk5 (12-14). These kinases are associated with neurotoxicity and implicated in neurodegenerative diseases. The JNK interacting protein (JIP) family possess phosphotyrosinebinding domains and interact with the YENPTY motif of APP, forming the APP-JIP-JNK complex (15). JIP-1 recruits multiple kinases in the JNK pathway and regulates constitutive axonal transport (16). Hence, we suspect that among the various kinases in AD conditions, JNK is involved in APP phosphorylation at Thr668.

To address these issues, treatments were carried out using several kinase inhibitors (including JNK inhibitor) to investigate whether phosphorylated APP at Thr668 were increased in OA-treated neurons; thereafter, we explored which pathway was involved in the phosphorylation of APP at Thr668. We found that phosphorylated APP at Thr668 was induced in OA-treated neurons, and the JNK inhibitor significantly suppressed phosphorylation of APP at Thr668 in a dosedependent manner.

\section{RESULTS}

Increased phosphorylation of APP at Thr668 in the axonal swellings of OA-treated neurons

In order to find out whether APP is also phosphorylated at

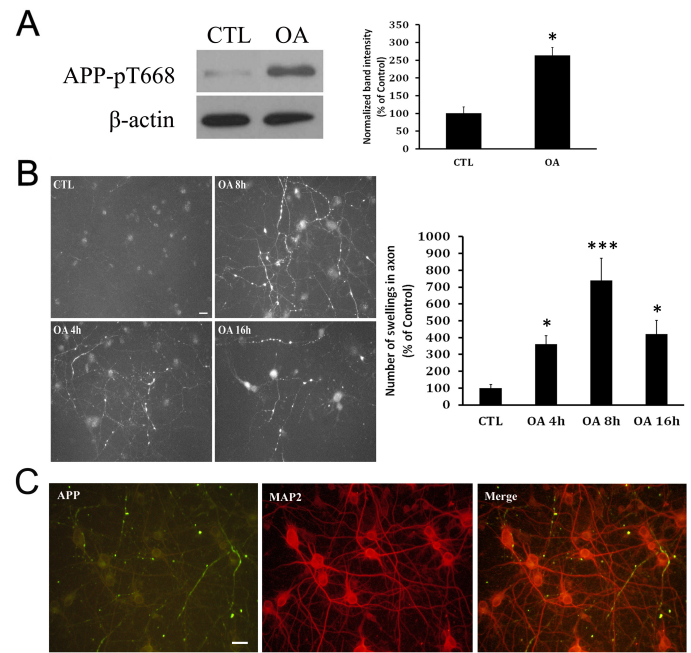

Fig. 1. Increased level of phosphorylated APP in OA-treated neuron cultures. (A) Western blot analysis with phosphorylated-amyloid precursor protein (APP) antibody shows that APP phosphorylation meaningfully increased in okadaic acid-treated neurons compared with control neurons. (B) Temporal changes in level of phosphorylated APP in the neurite swellings. Control neurons show reduced immunoreactivity for APP. APP immunoreactivity was increased in the neurite swellings within $4 \mathrm{hr}$ post treatment of OA; APP immunoreactivity was found to be accumulated in cell bodies at $16 \mathrm{hr}$ post-treatment. Scale bar represents $20 \mu \mathrm{m}$. (C) APP $(+)$ swellings in neurites does not colocalize with MAP2 $(+)$ dendrites. Scale bar represents $20 \mu \mathrm{m}$.
Thr668 in OA-treated neurons, we generated an AD cellular model (simulating AD brains), and the primary neuron cultures were immunostained with an antibody specifically detecting APP phosphorylated at Thr668. The neuronal extracts were analyzed by Western blotting. Interestingly, the intensity of band corresponding to phosphorylated APP at Thr668 in OA was about 2.5-fold higher than that in controls, 16 hours after treatment (Fig. 1A). As previously reported (17), APP was found to be accumulated in the microtubule-associated protein 2 (MAP2)-negative axons (Fig. 1C). The immunoreactivities for phosphorylated APP at Thr668 were robust in the axonal swellings of OA-treated neurons, in contrast to very weak signals in the neurites of control neurons (Fig. 1B).

\section{Colocalization of APP with JNK in the axonal swellings of OA-treated neurons}

We observed that JNK was activated in the OA-treated neurons (18). Since JNK is one of kinases to phosphorylate APP (19), we investigated whether JNK was involved in the phosphorylation of APP in OA-treated neurons. Primary neuron cultures were immunostained with antibodies detecting p-APP, p-JNK, JNK interacting protein (JIP)-1, and JIP-3. Interestingly, phosphorylated APP was co-localized with phosphorylated JNK (an active form of JNK) in the axonal swellings at 8 hours after OA treatment (Fig. 2A). Following the OA treatment, signals for phosphorylated APP at Thr668 were also co-localized with both JIP-1- and JIP-3-positive immunoreactivities in the axonal swellings (Fig. 2B and C).

\section{JNK inhibitor reduced the APP phosphorylation of OA-treated neurons}

In order to find out whether JNK phosphorylates APP in

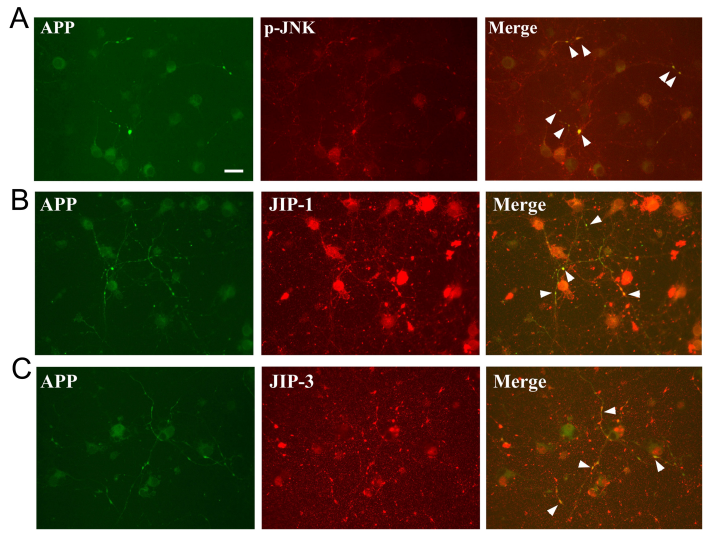

Fig. 2. Representative images showing colocalization of APP with JNK in the axonal swellings of OA-treated neurons. (A) p-JNK increases in the axonal swellings of OA-treated neurons, where APP immunoreactivity also increases (arrowheads) (B), (C) Similar to the consequences of $\mathrm{p}-\mathrm{JNK}$, both JIP-1 and JIP-3 indicates co-localization with APP phorphorylation in axonal swellings of OA-treated neurons. Scale bar represents $20 \mu \mathrm{m}$. 
OA-treated neurons, and whether other kinases including extracellular signal-regulated kinase (ERK), p38 mitogenactivated protein kinase (p38-MAPK), or cyclin-dependent kinase 5 (CDK5) also phosphorylates APP, primary neuron cultures were treated with various kinase inhibitors, such as a JNK inhibitor (SP600125) (20), an ERK inhibitor (PD98059), a p38-MAPK inhibitor (SB203580), and CDK5 inhibitors (olomoucine and roscovitine). Interestingly, only SP600125, a JNK inhibitor (but not other inhibitors), reduced the APP phosphorylation (Fig. 3A), indicating that JNK is the kinase for APP phosphorylation in OA-treated neurons. Furthermore, the degree of inhibition of APP phosphorylation at Thr668 was proportional to concentrations of the JNK inhibitor, in a dose-dependent manner (Fig. 3B).

\section{JNK inhibitor reduced the axonal accumulations of phosphorylated APP in OA-treated neurons}

Since APP phosphorylation at Thr668 was dramatically suppressed by JNK inhibitor, we next evaluated whether axonal accumulations of phosphorylated APP were reduced. As expected, SP600125 treatment decreased the immunoreactivity for phosphorylated APP at Thr668 in the axons of OA-treated neurons (Fig. 4A), implying the suppressive effects of JNK inhibitors on axonal accumulations of phosphorylated APP at Thr668. In order to investigate whether JNK was involved in OA-induced neurotoxicity, we pretreated neuronal cultures with SP600125 for 30 min before OA treatment, and evaluated the effects using an LDH-release assay. Treatment of neurons with $\mathrm{OA}$ for $48 \mathrm{hr}$ increased LDH release by about $150 \%$, as compared to control cultures. This OA-induced increase in $\mathrm{LDH}$ release was decreased by pretreatment with

\section{A}

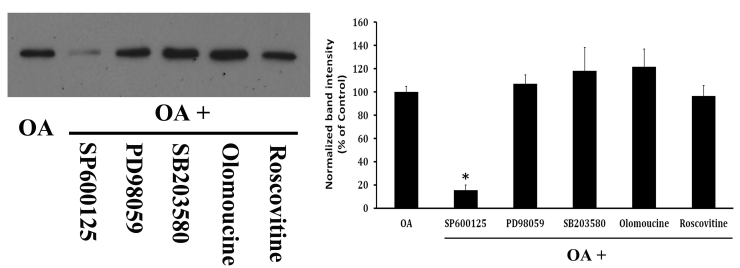

B

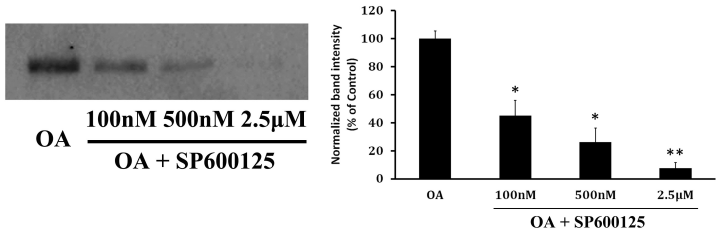

Fig. 3. Inhibition of OA-induced APP phosphorylation with various kinase inhibitors. (A) Western blot analysis with OA-treated neurons and several kinase inhibitors, including JNK inhibitor, shows that only JNK inhibitor-SP600125 meaningfully decreased APP phosphorylation. (B) Decrease of APP phosphorylation is generally proportional to concentration of JNK inhibitor.
SP600125 (Fig. 4B). This neuroprotective effect of SP600125 was further confirmed by the MTT assay. Treatment of neurons with OA for $48 \mathrm{hr}$ decreased MTT absorbance by about 30\%, compared to control cultures. This OA-induced decrease in MTT absorbance was reversed by pretreatment with SP600125 (Fig. 4B).

\section{DISCUSSION}

In the present study, we showed that phosphorylation of APP at Thr668 was increased in the axonal swelling of OA-treated neurons. The increase of phosphorylated APP (p-APP) and neurodegeneration was reduced by inhibition of JNK, suggesting that JNK is a therapeutic target in PP2A-inhibited neurodegenerative conditions, including AD.

Subcellular APP trafficking is a critical step for the accumulation of $A \beta$, the key determinant in pathogenesis of $A D$, and may be regulated by its phosphorylation (21). APP has eight potential phosphorylation sites in its cytoplasmic domain. Phosphorylation site Ser655 (numbering for human APP695 isoform) of ${ }^{653} \mathrm{YTSI}^{656}$ motif is expressed exclusively in the brain (22). The Thr668 site is predominantly involved in APP phosphorylation in neurons of the brain (12). Phosphorylation of APP at Thr668 (numbering for human APP695 isoform) is detectable in mature APP, but not in immature APP (12), indicating involvement of Thr668 in the late secretory pathway. APP is subject to $\mathrm{N}$-glycosylation (immature APP) in the endoplasmic reticulum and, during post-translational modifications, APP is modified with O-glycosylation in the Golgi complex. APP with both $\mathrm{N}$ - and O-glycosylation (mature
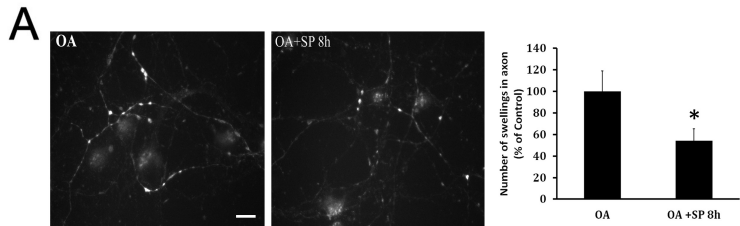

B
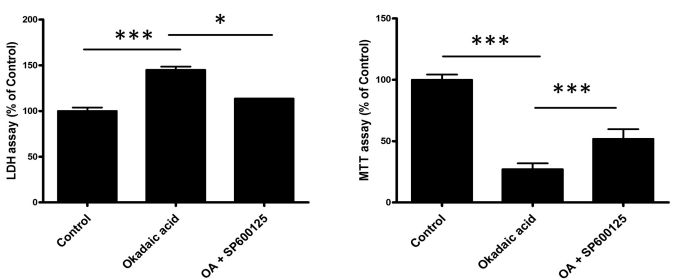

Fig. 4. Reduced level of phosphorylated APP with SP600125induced JNK inhibition. (A) APP immunoreactivity in OA-treated neuron at $8 \mathrm{~h}$ post treatment showed diminished axonal accumulations with SP600125 treatment. Scale bar represents $20 \mu \mathrm{m}$. (B) A quantitative $\mathrm{LDH}$-release assay showed that treatment with $\mathrm{OA}$ for $48 \mathrm{hr}$ increased LDH release by about $150 \%$ compared to untreated controls (CTL). Pretreatment with SP600125 reduced OA-induced $\mathrm{LDH}$ release by about $50 \%\left({ }^{*} \mathrm{P}<0.05\right.$ vs. OA). MTT assay also shows a protective effect of SP600125. 
APP) localizes to the late protein secretory pathway, in which mature APP is cleaved by secretases (23). Mature APP undergoes further modification by phosphorylation, also occurring in the late secretory pathway.

It has been reported that APP and APP- $\beta C T F$ phosphorylation at Thr668 is increased in AD brains; the mutation of Thr668 (replacement with alanine) significantly reduced $A \beta$ generation (less than 50\%), suggesting that the Thr668 phosphorylation site may be pivotal to facilitate $\beta$-secretase cleavage of APP and generation of A $\beta$ (10). Indeed, this APP phosphorylation was not found significantly increased in other neurodegenerative diseases, including Huntington's disease, Parkinson's disease, Progressive Supranuclear Palsy, and Frontal Temporal Dementia, suggesting that APP phosphorylation at Thr668 is pathology specific to AD. However, limitations exist in the mouse model study (11). Mutant mice possessing alanine substitution for Thr668 of APP did not show any significant differences in APP metabolism, including $A \beta$ generation, when compared to the wild-type animals. Nevertheless, compensatory effects cannot be excluded. Besides, contrary to this observation, substantial reports have supported that phosphorylation of APP at Thr668, or amino acid substitutions mimicking phosphorylated APP at Thr668, regulates the cleavage by $\gamma$-secretase in vitro $(10,13,14,24,25)$.

Consistent with previous reports, OA treatment induced phosphorylation of APP- $\beta$ CTF at Thr668 in the axonal swellings of primary cortical neurons in the present study, as shown in the brain of AD patients. Thr668 phosphorylation site also can be activated by various protein kinases, such as GSK3 $\beta$, SAPK1b/JNK3, and Cdk5 (12-14). These kinases are associated with neurotoxicity and implicated in neurodegenerative diseases. Thr668 phosphorylation results in significant conformational changes affecting the interactions of APP with its binding partners. In addition to these kinases, phosphorylation of APP and $\beta$ CTF at Thr668 can be regulated by c-jun $\mathrm{N}$-terminal kinases (JNK). Many enzymes are involved in the phosphorylation of APP in the AD brain, which remain unclear. Results in this study suggest one possibility that JNK may play a central and more significant role than other kinases in the phosphorylation of APP at Thr688 in PP2A-inhibited neurodegenerative conditions, since the inhibitors targeting other kinases could not reduce the phosphorylation of APP (Fig. 3). Moreover, JNK inhibitor decreased the axonal accumulation of phosphorylated APP and neurotoxicity (Fig. 4).

According to the p-JNK recruitment in axonal swellings, JIP-1 and JIP-3 are also highly co-localized with APP- $\beta C T F$, which means that they form APP-JIP-JNK complex (15). JIP family possesses phosphotyrosine-binding domains and interacts with the YENPTY motif of APP. JIP-1 recruits multiple kinases in the JNK pathway and regulates constitutive axonal transport (16). JIP-1b may function as a scaffold between APP and JNK, and may also link APP and kinesin light chain-1. Conversely, JIP-3 is a structurally unrelated scaffolding protein (26). It seems to play a role in injury signaling (27), which is evidenced by the retrograde axonal transport of $\mathrm{p}$-JNK and JIP found in ligated sciatic nerve, suggesting a mechanism in sensing and repairing the abnormal state at the periphery, far from the cytosol.

In addition to the JIP family, there are a number of APP interacting proteins, some of which were observed in our previous experiments. For example, the $\beta$ - and $\gamma$-secretases of APP are fascinating targets for drug in treating AD. However, secretase inhibitors may have intolerable toxicities and side effects, since APP is widely expressed through tissues and the inhibitors can block general beneficial functions of APP. Accordingly, another approach to AD therapeutics based on the amyloid hypothesis can be provided by regulatory APP adaptor proteins because it can serve as more specific targets. Obviously, more studies are required to discover which interacting proteins are more functionally relevant to $A D$ pathogenesis. The OA-induced neurodegeneration model may serve as a useful tool for studying the AD pathogenesis.

SP600125 is a selective and reversible inhibitor of JNK (20). Furthermore, we found activated JNK is almost completely co-localized with accumulated APP-BCTFs in axonal swellings, implicating that JNK might be a major kinase for APP phosphorylation in OA-induced neurodegeneration. As OA induces neuronal damage with GSK3 $\beta$ activation (8) and hyperphosphorylation (a characteristic feature of AD pathology), this study indicates that JNK related APP processing and its inhibition could be important in the pathophysiology and therapeutics of Alzheimer's disease.

Taken together, we herein provided the possible protective effects of reducing the phosphorylation by JNK specific inhibitors, in OA-treated neurons. Phosphorylation in OAtreated neurons decreased with higher concentration of SP600125. As the JNK/C-Jun cascade can be activated in the $\mathrm{AD}$ brain and was toxic to neurons, these findings provide important insights into JNK down-regulation and OA-induced neurodegeneration that may be extended into studies on the pathogenesis of $\mathrm{AD}$.

\section{MATERIALS AND METHODS}

\section{Neuron culture}

Primary cortical neuron cultures were prepared from the brains of embryonic day-16 rat pups, as previously described $(28,29)$. Briefly, the cerebral cortices were dissected in calcium- and magnesium-free Hank's balanced salt solution, and incubated in a $0.125 \%$ trypsin solution for $10 \mathrm{~min}$ at $37^{\circ} \mathrm{C}$. Trypsin was inactivated with Dulbecco's modified Eagle's medium (DMEM) containing 20\% fetal bovine serum, and the cortical tissue was further dissociated by serial trituration using a Pasteur pipette. The resulting cell suspensions were diluted in neurobasal medium supplemented with B27 components (GibcoBRL) and plated onto polyD-lysine- (Sigma, $50 \mu \mathrm{g} / \mathrm{ml}$ ) and laminin-coated $(1 \mu \mathrm{g} / \mathrm{ml}$, GibcoBRL) 48 -well plates at a density of $5 \times 10^{4}$ cells per 
well. Neurons were maintained at $37^{\circ} \mathrm{C}$ in a $5 \%$ CO2 atmosphere for 12 days. Cells were pretreated with various inhibitors, including SP600125 (Tocris Bioscience, Ellisville, MO), PD98059 (Calbiochem, San Diego, CA), SB203580 (Calbiochem), Olomoucine (Sigma-Aldrich), and Roscovitine (Calbiochem), prior to the addition of OA (10 nM, SigmaAldrich).

\section{Western blot analysis}

For Western blot analysis, cultures were scraped with a cell-scraper and centrifuged at 2,000 rpm for $5 \mathrm{~min}$. Cell pellets were re-suspended in protein extraction solution (Pro-Prep, Intron) according to the manufacturer's guidelines, and incubated at $-20^{\circ} \mathrm{C}$ for $20 \mathrm{~min}$. The samples were then centrifuged at $13,000 \mathrm{rpm}\left(4^{\circ} \mathrm{C}\right)$ for $5 \mathrm{~min}$ and the supernatants were transferred to $1.5-\mathrm{ml}$ tubes. Protein concentrations were measured by the Bradford method. Equal amounts of protein were mixed with sample buffer [62.5 mM Tris- $\mathrm{HCl}(\mathrm{pH} 6.8)$, $1 \%$ sodium dodecyl sulfate (SDS), $2.5 \%$ glycerol, $0.5 \%$ $\beta$-mercaptoethanol, and bromophenol blue], boiled at $100^{\circ} \mathrm{C}$ for $5 \mathrm{~min}$, and stored at $-20^{\circ} \mathrm{C}$ until use. Proteins were subjected to SDS-polyacrylamide gel electrophoresis at a constant voltage $(130 \mathrm{~V})$ and were subsequently transferred to polyvinylidene difluoride membranes (pore size, $0.2 \mathrm{~mm}$; Biorad) at $110 \mathrm{~V}$ for $2 \mathrm{~h}$. The blots were then incubated for $1 \mathrm{~h}$ in a blocking TTBS buffer $(20 \mathrm{mM}$ Tris, $137 \mathrm{mM} \mathrm{NaCl}, 0.1 \%$ Tween-20) containing $2 \%$ bovine serum albumin (BSA) with $2 \%$ normal goat serum, and following which they were incubated with phosphor-APP (Thr668) antibody (1:1000, Cell Signaling) or $\beta$-actin $\left(1: 2000\right.$, Sigma) for $16 \mathrm{~h}$ at $4^{\circ} \mathrm{C}$. Finally, the blots were washed with TTBS buffer, incubated with HRP-labeled anti-lgG antibody (1:5,000, Pierce), and then visualized using enhanced chemiluminescence reagents (Amersham) and x-ray film.

\section{Immunocytochemistry}

For immunofluorescence investigations, cells grown on glass coverslips were fixed for 30 minutes at room temperature in $4 \%$ paraformaldehyde in $0.1 \mathrm{M}$ phosphate buffer (30). Cell membranes were permeabilized by incubation for 30 minutes in $0.05 \mathrm{M}$ Tris buffer $(\mathrm{pH} 7.4)$ containing $0.1 \%$ Triton X-100, $2 \% \mathrm{BSA}$, and $2 \%$ normal goat serum. Cultures were incubated overnight at $4^{\circ} \mathrm{C}$ in primary antibody solution containing either phosphor-APP (Thr668) antibody (1:500, Cell Signaling), MAP2 antibody (1:500, EMD Millipore), p-JNK antibody (1:500, Cell Signaling), JIP-1 antibody (1:50, Santa Cruz), or JIP-3 antibody (1:50, Santa Cruz). Cells were then washed, followed by incubation with fluorescein-isothiocyanate (FITC)- or Cy3labeled secondary antiserum (1:300, Jackson Laboratories).

\section{LDH assay}

After $48 \mathrm{hr}$ of treatment, cell injury in neuron cultures was quantitatively assessed by measuring the lactate dehydrogenase $(\mathrm{LDH})$ activity released into the bathing medium from damaged cells, using a Cytotox 96 assay kit (Promega), according to the manufacturer's guidelines (31).

\section{MTT assay}

After $48 \mathrm{hr}$ of treatment, neuronal cell viability was assessed using the 3-(4,5-dimethylthiazol-2-yl)-5-(3-carboxymethoxyphenyl)-2-(4-sulfophenyl)-2H-tetrazolium (MTS) reduction assay (CellTiter kit, Promega), as instructed by the manufacturers. Absorbance was measured spectrophotometrically at $490 \mathrm{~nm}$.

\section{Statistical analysis}

The data are presented as means \pm s.e.m. from three independent experiments, each performed in duplicate. The data were analyzed using the Student's t-test, or one way ANOVA followed by Tukey post-hoc test. $\mathrm{P}<0.05$ was considered statistically significant.

\section{ACKNOWLEDGEMENTS}

This work was supported by the Medical Research Center Program through the National Research Foundation of Korea (NRF) funded by the Ministry of Science, ICT \& Future Planning (2008-0062286), and by a grant of the Korean Health Technology R\&D Project, Ministry of Health \& Welfare, Republic of Korea (HI13C1630).

\section{REFERENCES}

1. Gomez-Isla T, Hollister R, West $\mathrm{H}$ et al (1997) Neuronal loss correlates with but exceeds neurofibrillary tangles in Alzheimer's disease. Ann Neurol 41, 17-24

2. Hardy J and Selkoe DJ (2002) The amyloid hypothesis of Alzheimer's disease: progress and problems on the road to therapeutics. Science 297, 353-356

3. Grundke-lqbal I, Iqbal K, Tung YC, Quinlan M, Wisniewski $\mathrm{HM}$ and Binder LI (1986) Abnormal phosphorylation of the microtubule-associated protein tau (tau) in Alzheimer cytoskeletal pathology. Proc Natl Acad Sci U S A 83, 4913-4917

4. Tanimukai H, Grundke-lqbal I and lqbal K (2005) Up-regulation of inhibitors of protein phosphatase-2A in Alzheimer's disease. Am J Pathol 166, 1761-1771

5. Tachibana K, Scheuer PJ, Tsukitani Y et al (1981) Okadaic acid, a cytotoxic polyether from two marine sponges of the genus Halichondria. J Am Chem Soc 103, 2469-2471

6. Arias C, Sharma N, Davies P and Shafit-Zagardo B (1993) Okadaic acid induces early changes in microtubuleassociated protein 2 and tau phosphorylation prior to neurodegeneration in cultured cortical neurons. J Neurochem 61, 673-682

7. Sun L, Liu SY, Zhou XW et al (2003) Inhibition of protein phosphatase $2 \mathrm{~A}$ - and protein phosphatase 1 -induced tau hyperphosphorylation and impairment of spatial memory retention in rats. Neuroscience 118, 1175-1182

8. Kamat PK, Rai S and Nath C (2013) Okadaic acid induced neurotoxicity: an emerging tool to study Alzheimer's 
disease pathology. Neurotoxicology 37, 163-172

9. Wang Z, Yang L and Zheng H (2012) Role of APP and Abeta in synaptic physiology. Curr Alzheimer Res 9, 217-226

10. Lee MS, Kao SC, Lemere CA et al (2003) APP processing is regulated by cytoplasmic phosphorylation. J Cell Biol $163,83-95$

11. Sano Y, Nakaya T, Pedrini $S$ et al (2006) Physiological mouse brain Abeta levels are not related to the phosphorylation state of threonine-668 of Alzheimer's APP. PLoS ONE 1, e51

12. lijima K, Ando K, Takeda S et al (2000) Neuron-specific phosphorylation of Alzheimer's beta-amyloid precursor protein by cyclin-dependent kinase 5. J Neurochem 75, 1085-1091

13. Liu F, Su Y, Li B et al (2003) Regulation of amyloid precursor protein (APP) phosphorylation and processing by p35/Cdk5 and p25/Cdk5. FEBS Lett 547, 193-196

14. Rockenstein E, Torrance M, Adame A et al (2007) Neuroprotective effects of regulators of the glycogen synthase kinase-3beta signaling pathway in a transgenic model of Alzheimer's disease are associated with reduced amyloid precursor protein phosphorylation. J Neurosci 27, 1981-1991

15. Muresan Z and Muresan V (2005) c-Jun NH2-terminal kinase-interacting protein-3 facilitates phosphorylation and controls localization of amyloid-beta precursor protein. J Neurosci 25, 3741-3751

16. Dickens M, Rogers JS, Cavanagh J et al (1997) A cytoplasmic inhibitor of the JNK signal transduction pathway. Science 277, 693-696

17. Yoon SY, Choi JE, Yoon JH, Huh JW and Kim DH (2006) BACE inhibitor reduces APP-beta-C-terminal fragment accumulation in axonal swellings of okadaic acid-induced neurodegeneration. Neurobiol Dis 22, 435-444

18. Yoon S, Choi J, Yoon J, Huh JW and Kim D (2006) Okadaic acid induces JNK activation, bim overexpression and mitochondrial dysfunction in cultured rat cortical neurons. Neurosci Lett 394, 190-195

19. Inomata H, Nakamura $Y$, Hayakawa A et al (2003) A scaffold protein JIP-1b enhances amyloid precursor protein phosphorylation by JNK and its association with kinesin light chain 1. J Biol Chem 278, 22946-22955

20. Bennett BL, Sasaki DT, Murray BW et al (2001) SP600125 an anthrapyrazolone inhibitor of Jun $\mathrm{N}$-terminal kinase. Proc Natl Acad Sci U S A 98, 13681-13686

21. Jiang S, Li Y, Zhang X, Bu G, Xu H and Zhang YW (2014) Trafficking regulation of proteins in Alzheimer's disease. Mol Neurodegener 9, 6

22. Kang J, Lemaire HG, Unterbeck A et al (1987) The precursor of Alzheimer's disease amyloid A4 protein resembles a cell-surface receptor. Nature 325, 733-736

23. Suzuki $T$ and Nakaya $T$ (2008) Regulation of amyloid beta-protein precursor by phosphorylation and protein interactions. J Biol Chem 283, 29633-29637

24. Pastorino L, Sun A, Lu PJ et al (2006) The prolyl isomerase Pin1 regulates amyloid precursor protein processing and amyloid-beta production. Nature 440, 528-534

25. Feyt C, Pierrot N, Tasiaux B et al (2007) Phosphorylation of APP695 at Thr668 decreases gamma-cleavage and extracellular Abeta. Biochem Biophys Res Commun 357, 1004-1010

26. Whitmarsh AJ (2006) The JIP family of MAPK scaffold proteins. Biochem Soc Trans 34, 828-832

27. Abe N, Almenar-Queralt A, Lillo C et al (2009) Sunday driver interacts with two distinct classes of axonal organelles. J Biol Chem 284, 34628-34639

28. Cho K, Cho MH, Seo JH et al (2015) Calpain-mediated cleavage of DARPP-32 in Alzheimer's disease. Aging Cell $14,878-886$

29. Song HL, Shim S, Kim DH et al (2014) beta-Amyloid is transmitted via neuronal connections along axonal membranes. Ann Neurol 75, 88-97

30. Park JS, Kim DH and Yoon SY (2016) Regulation of amyloid precursor protein processing by its KFERQ motif. BMB Rep [Epub ahead of print]

31. Lee JH, Kim IW, Kim SH et al (2015) Anticancer activity of CopA3 dimer peptide in human gastric cancer cells. BMB Rep 48, 324-329 